\title{
Fundamental study on spontaneous corrugation pattern on dry sand due to moving vehicle
}

\author{
Aiko Ikeda ${ }^{1, *}$, Kai Hashimoto ${ }^{1}$, Teeranai Srimahachota ${ }^{1}$, Hao Zheng ${ }^{2}$, and Shunji Kanie ${ }^{2}$ \\ ${ }^{1}$ Graduate School of Engineering, Hokkaido University, Sapporo, Japan \\ ${ }^{2}$ Fucalty of Engineering, Hokkaido University, Sapporo, Japan
}

\begin{abstract}
On unpaved or snowy icy roads, it is known that corrugation pattern on road surface spontaneously emerges due to the repetition of loading by vehicle, and this phenomenon is named washboard road. Washboard road on snowy icy road causes serious problem on the driving of vehicles because the braking performance deteriorates due to lack of friction between road surface and tires. The authors developed an indoor experimental device to reproduce similar phenomenon in a laboratory. As the first step of the study, we chose fine dry sand as a surface material and we successfully reproduced the corrugation on the track. After scrutinizing the growing process of corrugation, we found that the running velocity of the track and the natural frequency of the oscillator are the most important factors for the growth. However, the frequencies of the corrugation converge to some specific value which is different from the natural frequency of the oscillator. Further, we adopted different road materials such as saturated sand, artificial snow and natural snow. This paper introduces interesting findings on formation process of washboard road.
\end{abstract}

\section{Introduction}

It is known that corrugation pattern on road surface of unpaved or snowy icy road spontaneously emerges due to the repetition of loading by vehicle [1]. Similar corrugation pattern also occurs on farmland when agricultural tractor drags plow or hoe. They are called as washboard road in English or Soroban road in Japanese. In winter Hokkaido, Japan, the washboard road on snowy icy road causes serious problem on the driving of vehicles because the braking performance deteriorates due to lack of friction between road surface and tires. At the same time, it endangers pedestrians on crossing walks and deteriorates the comfortability of vehicle passengers. In order to maintain the safe road condition during winter, it is essential to clarify the formation mechanism of this phenomenon.

\subsection{Observation Study for Washboard Road}

\subsubsection{Corrugation on Snowy lcy Roads}

In Hokkaido, it has been confirmed that irregular corrugation of ice is likely to occur where vehicles frequently decelerate or stop, such as intersections in winter (Fig. 1.). The occurrence of washboard road becomes frequently observed after the prohibition in use of studded tires. Matsukura et al. reported the formation process of corrugation on snowy icy roads as follows [2]. First, snow fell on road surface forms slight corrugation, and it gradually changes to a rippled thin snow layers. In this process, the convex of the ripple keeps growing at the same position and never moves in back or forward directions regardless with thawing and additional snowfall. However, the rippled surface suddenly changes to washboard road and Matsukura explained the seeping of thawed water into the bottom of snowy icy layer as the reason of this change. On the other hand, Kanie et al. found essential conditions in which washboard road emerges and grows [3]. Based on the fixed point observation by time-lapse cameras, they advocate the following three conditions should be satisfied simultaneously. That is; 1 . Sufficient amount of traffic, 2 . Ample of snow fall in thawing phase and 3. The air temperature between 0 and -2 degrees. However, the formation process of washboard road has not yet been clarified theoretically and scientifically.

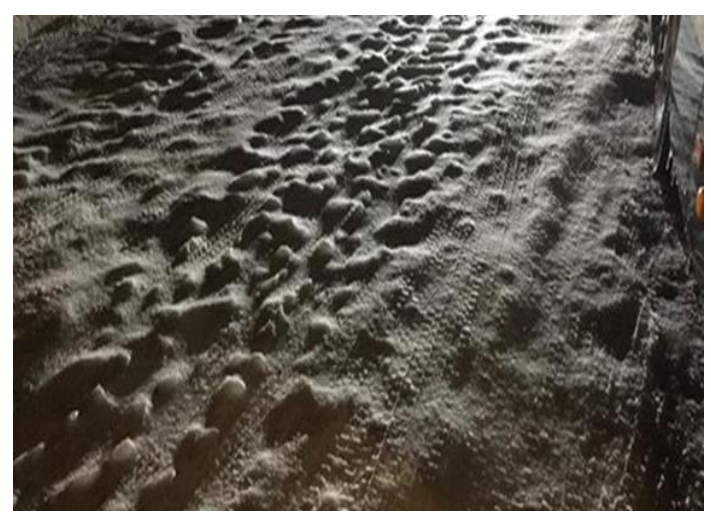

Fig. 1. Corrugation on Snowy Icy Road

* Corresponding author: aaaik_rm@eis.hokudai.ac.jp 


\subsubsection{Corrugation on Unpaved Roads}

On unpaved road with dry sand and gravel, it has been broadly known that washboard road is spontaneously formed by traffic vehicles (Fig. 2.) and several papers have explained the phenomenon. One of those says that the washboard road is likely to occur when the surface material is in dry condition, for example. Then, corrugation on unpaved road is frequently observed during summer because moisture in soil easily evaporates into the air. Another researcher, Takeshita, considers the causes from both of external and internal factors [4]. As an external factor, he indicated the natural frequency of vehicle decided by the combination of spring coefficient and damping performance equipped in the suspension of vehicle. As an internal factor, on the other hand, material properties of surface are referred. Whereas cohesive moist clay is not likely to scatter, dry and fine sand is easily rolled up and blown off by the centrifuge of rotating wheel. The mobility of surface material is thought as an important factor of the formation.

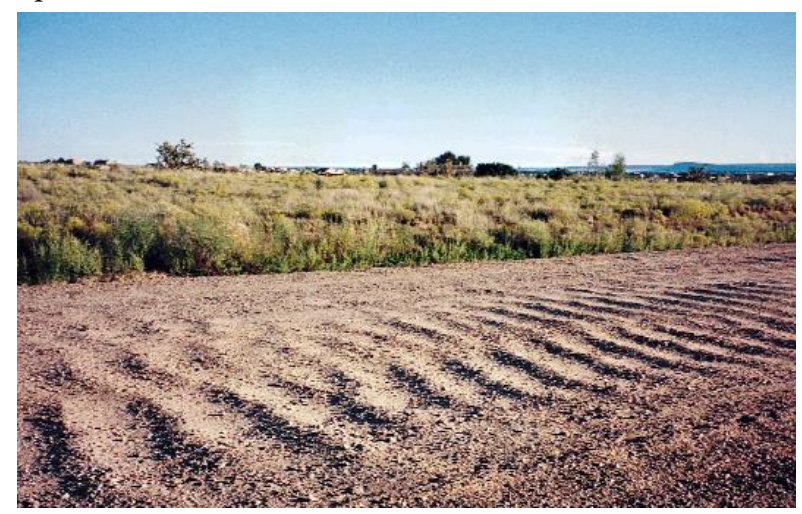

Fig. 2. Corrugation on Unpaved Road [5]

\subsection{The Purpose of This Research}

Since the formation mechanism of spontaneous corrugation pattern on road surface due to moving vehicles has not yet been clarified, the authors adopted an empirical approach with an easily available material such as sand to reproduce the phenomenon which occurs on unpaved road [6]. This research aims to explain the formation mechanism of washboard road by a scientific approach, and to propose a forecasting method of occurrence as well as effective countermeasures. In this paper, we focus on the results and discussion mainly based on the experimental approach with dry sand as a road material while changing environmental factors. In addition, we carried out a penetration test to obtain the relationship between the downward force due to wheel and the depth of penetration in order to confirm the deformation ability of the sand. The flow chart of this study is as shown in Fig. 3. The authors believe that the research finally contributes to propose some new management technology for safe and reliable road network.

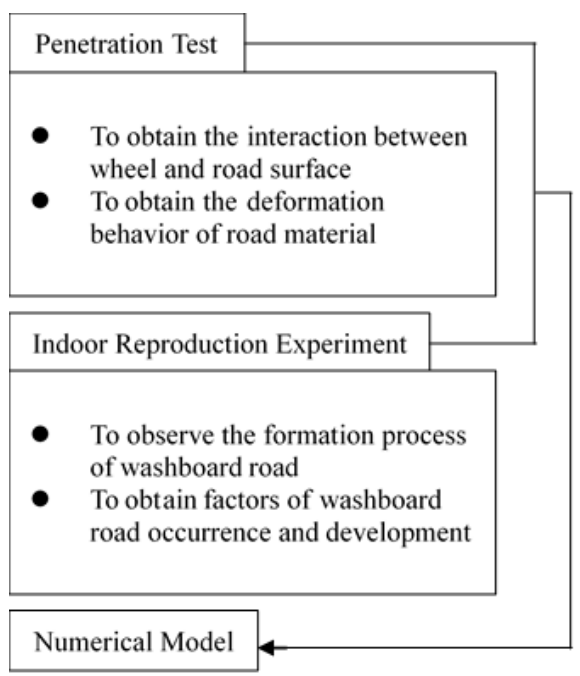

Fig. 3. Flowchart of This Research

\section{Penetration Test}

Due to some action of force transmitted by wheel, the sand surface gradually deforms and migrates. As a result, it finally causes the formation of washboard road. Then, it is obviously important to know the deformation ability of sand material due to the interaction between the wheel and the surface material. The authors provided a penetration test device to confirm the deformation ability of sand named as a penetration test $[7,8]$.

\subsection{Experimental Setup}

Fig. 4. shows the penetration test device. The attachment modelling a wheel is gradually settled down into the test material, and the penetration depth is simultaneously measured by a laser displacement sensor. The magnitude of force transmitted to the material through the attachment is recorded by an electronic balance installed at the bottom of the material. The downward force magnitude is controlled by the pendulums put at the top, and the maximum load applied to the material is approximately 5 $\mathrm{N}$. By repeating the loading and unloading three times, we investigated the relationship between the penetration depth and the magnitude of force. The attachment modelling a wheel is in a shape of cylinder made of acrylonitrile butadiene styrene plastic with $3 \mathrm{~cm}$ in diameter and $1.5 \mathrm{~cm}$ in width. As a fine sand material, we used Toyoura standard sand, which is a silica sand designated by JIS (Japanese Industrial Standard) [9]. In the loading phase, a displacement-control procedure is adopted by increasing the vertical displacement of $0.1 \mathrm{~mm}$ at each step, and the corresponding load magnitude is recorded. When the attachment penetrates to the deepest position under the maximum load, the loading phase is terminated. In the unloading phase, on the contrary, a displacement-control procedure cannot be applied because the deformation of sand surface has already been in inelastic range. Then, a load-control procedure is employed by decreasing the loading magnitude of $0.1 \mathrm{~N}$ at each step, and the corresponding height of the attachment is observed. The unloading is completed when the loading magnitude shows no changes around $0 \mathrm{~N}$. 
These loading and unloading operations were repeated three times.

\subsection{Experimental Results}

Fig. 5. shows the relationship between the penetration depth and the loading magnitude obtained through the penetration test. In this figure, the experimental results for three cycles of loading and unloading are illustrated for comparison. However, as mentioned above, the sand surface has already been deformed in inelastic range. Then, the displacement of attachment is defined as $0 \mathrm{~mm}$ in the horizontal axis when it contacts with the sand surface again in the second and third cycles. In the loading phase, the displacement linearly increases with the force magnitude regardless of the repetition. However, the increment in the displacement for the first cycle is much larger than those of second and third cycles because the surface material has been sufficiently compacted and migrated during the first cycle, and the deformation ability of the material is lessen. In the unloading phase, the displacement shows a slight recovery from the deepest position, and this fact can be thought as another evidence for that the material has been deformed in plastic region.

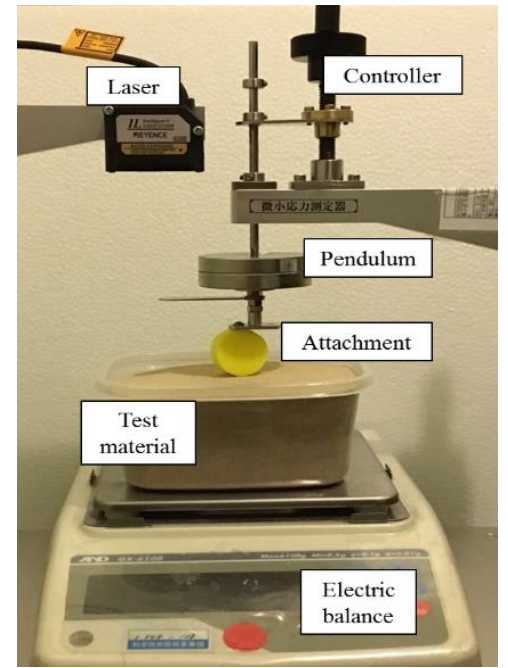

Fig. 4. Penetration Test Instrument

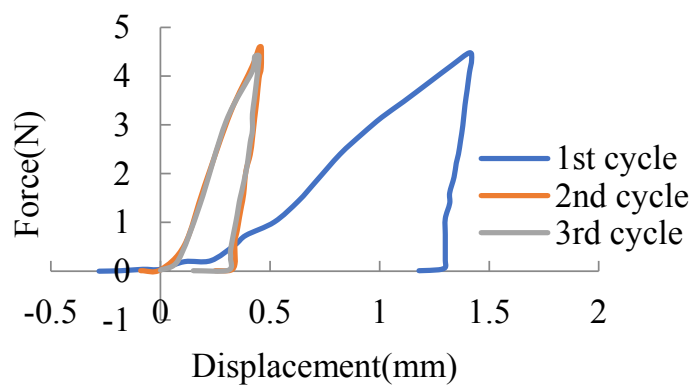

Fig. 5. Relationship between Displacement and Force

\subsection{Difference of the Shape of the Attachment in Penetration Test}

As a next step, we tried to evaluate the shape effect of the attachment on the penetration depth. Therefore, we prepared several different shapes of the attachments in addition to the cylindrical one (Fig. 6.). Three attachments in triangular shape have different tip angles of 60,90 and 120 degrees with the same thickness of $1.5 \mathrm{~cm}$. A hemisphere is another type of attachment with a diameter of $3.0 \mathrm{~cm}$. All the attachments are made of acrylonitrile butadiene styrene plastic.

Fig. 7. shows the experimental results of the relationship between the penetration depth and the force magnitude in their first cycles. It can be seen that the gradient of the graph in the first cycle differs with the shape of attachment. For example, the triangular one with a tip angle of 60 degrees is easily settled down with the least force magnitude. However the tip angle becomes larger from 60 to 120 degrees, greater force is necessary to obtain a same penetration depth. In the case of cylindrical one, the gradient is steeper than any of those in triangular. Those results seem very natural because the steepness in the tip angle has a significant effect on the penetration ability. Comparing the gradients of the cylindrical and hemisphere ones, we also found clear difference between those, and the difference is caused by the shape effect in the transvers direction to the driving track. From those results, it can be concluded that the smaller the contact angle between the attachment and the horizontal surface becomes, the larger the force magnitude to induce a same penetration is.

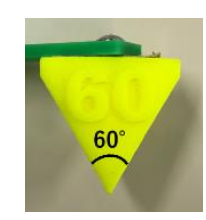

Triangular $\left(60^{\circ}\right)$

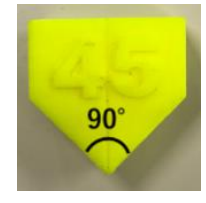

Triangular $\left(90^{\circ}\right)$

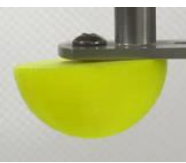

Hemisphere
Fig. 6. Attachment Shape Type

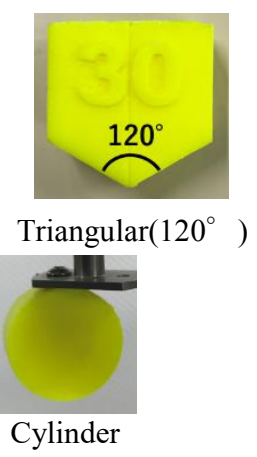

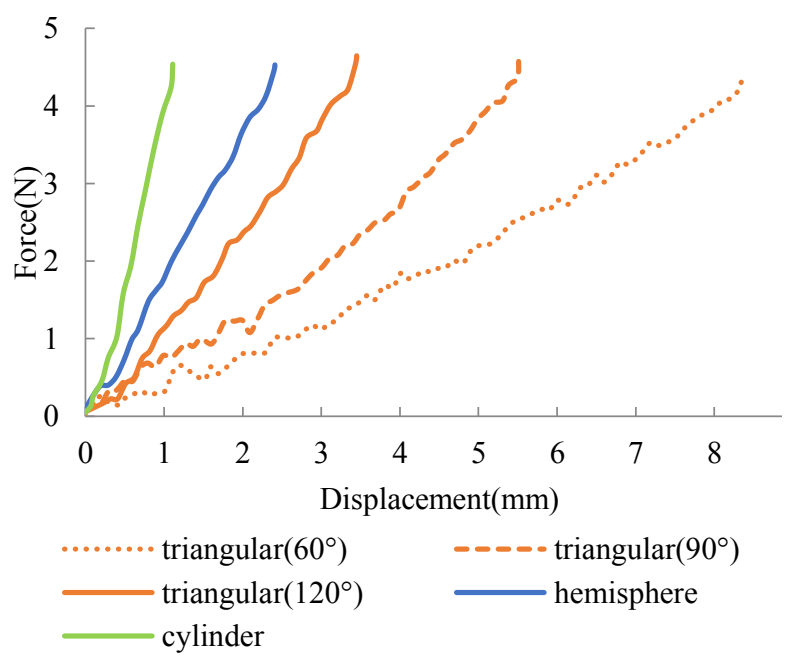

Fig. 7. Relationship between Displacement and Force in All Shape Type Attachments 


\section{Indoor Reproduction Experiment}

It is known that washboard road spontaneously emerges when some specific conditions are satisfied. However, the conditions necessary for the occurrence have not yet been understood, and the authors tried to observe the formation process including transient phase by an indoor reproduction experiment. After learning by trial and error, we successfully developed our original experimental apparatus which consists of a self-rotating track and a seesaw-shaped oscillator.

\subsection{Experimental Setup}

\subsubsection{Self-Rotating Track}

Fig. 8.(a) shows the experimental apparatus. It consists of a self-rotating track and a seesaw-shaped oscillator equipped with a laser displacement sensor. The outer and inner boundaries of the track are $50 \mathrm{~cm}$ and $41 \mathrm{~cm}$ in diameter respectively and the gap between two circular boundaries is filled with surface material. As our standard experimental condition, we adopted dry granular sand, called as Toyoura sand regulated by Japanese Industrial Standard (JIS), with an average particle size of $0.2 \mathrm{~mm}$. The rotation velocity of the track can be tuned from 0 up to 20.0 rounds per minute (rpm) precisely, and the maximum value in the relative speed of the rotating track corresponds to $0.5 \mathrm{~m} / \mathrm{s}$. The seesaw-shaped oscillator is fixed at the end of a cantilever beam extending from the rotational axis of the track as showing in Fig. 8.(b). The time-dependent change in height of the surface material is observed by a laser displacement sensor set just behind the oscillator. The sampling frequency of the sensor is 100 $\mathrm{Hz}$ with an accuracy of $1 \mu \mathrm{m}$ in height. The data obtained was used to analyze the geometrical feature of the corrugated pattern with time-dependent evolution throughout an experiment [10-12].

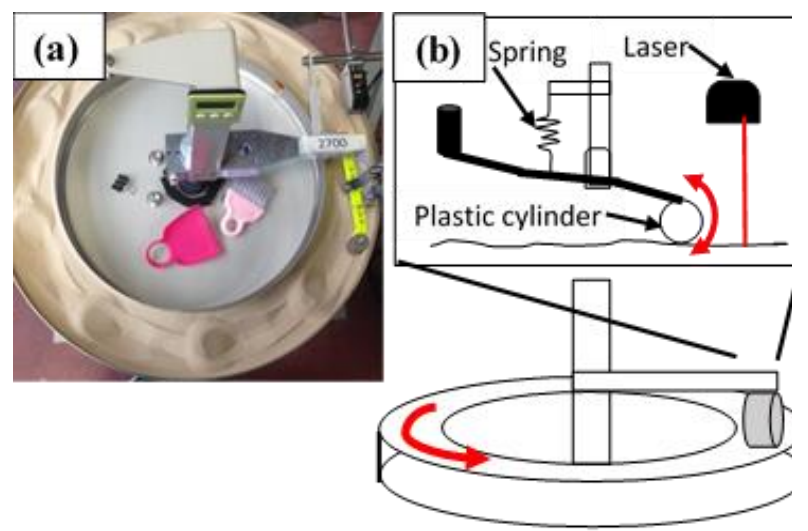

Fig. 8. Self-Rotating Track

\subsubsection{Seesaw-Shaped Oscillator}

The seesaw-shaped oscillator plays a key role in our reproduction experiments. The total length of the arm is $14.2 \mathrm{~cm}$, and one of the same attachments used for the penetration test is mounted at one end of the arm. In this experiment, the attachments do not roll unlike a real wheel of vehicle, and are simply dragged on the road surface. On the other hand, pendulum is loaded at the other end as a counterweight to control the natural frequency of the oscillator. The middle part of the seesaw arm is mechanically suspended by a soft spring which promotes the vertical oscillation of the attachment. The natural frequency of oscillation can be decided by giving an appropriate counterweight, as explained later. Before revolving the rotating track, we set the oscillator to be in balance of force while the bottom edge of the attachment gently touches the flat surface of the road material with almost no compressive force against the surface. The oscillator vibrates freely under keeping the balance in forces even after the track starts revolving.

At the beginning, the self-rotating track revolves at a slow speed of $3 \mathrm{rpm}$ for 2 minutes, and it is confirmed that the oscillator keeps same position without vibration on the flat surface. Then, the rotating velocity is increased to an experimental value without stopping the rotation, and the track keeps revolving continuously for 7 minutes at least. The change in height of the road surface is measured with a laser displacement sensor. The recorded data is analyzed by using Fast Fourier Transform to investigate the amplitude with its wave length of the corrugation after the experiment.

In this experimental procedure, the most difficult is to make the road surface flat as its initial condition. During the first 2 minutes with a slow rotating velocity of $3 \mathrm{rpm}$, it is important to confirm that no vibration of oscillator occurs. In all experimental cases, the oscillator slightly moved in vertical direction in accordance with the road surface but the vertical motion is fully repeated for the first 2 minutes to show the original corrugation of the surface. The authors assessed by this fact that the initial corrugation on the surface is infinitesimal small and negligible.

\subsection{Experimental Results}

\subsubsection{Reproduction Experiment on Dry Sand}

Experiments were conducted with the track rotation velocity increased from $9 \mathrm{rpm}$ to $19.5 \mathrm{rpm}$ incrementally by $1.5 \mathrm{rpm}$. Table 1. shows the number of waves formed on the track at the end of each experiment, the maximum amplitude of the road surface and the frequency of the road surface. We used an oscillator with a natural frequency of $1.66 \mathrm{~Hz}$ in these experiments. By keeping the experimental velocity of rotation, the road surface gradually waves from the initial flat condition, and we found that the faster the track rotation velocity is, the earlier the time to start waving is and the more it grows. As the track rotation velocity increases, waves with large wave length and amplitude are formed on the track at the end of the experiment, and the number of waves decreases. Interesting is that the wave frequency of the road surface at the maximum amplitude is almost constant as approximately $2.3 \mathrm{~Hz}$ to $2.4 \mathrm{~Hz}$ and it is larger than the natural frequency of the oscillator. From those, it can be seen that the occurrence of corrugation is greatly depending on the track rotating velocity. 
Table 1. Comparison of Experimental Corrugation

\begin{tabular}{c|c|c|c}
\hline $\begin{array}{c}\text { Velocity } \\
(\mathrm{rpm})\end{array}$ & $\begin{array}{c}\text { Wave } \\
\text { Number }\end{array}$ & $\begin{array}{c}\text { Max } \\
\text { Amplitude } \\
(\mathrm{mm})\end{array}$ & $\begin{array}{c}\text { Finish } \\
\text { Peak } \\
\text { Frequency } \\
(\mathrm{Hz})\end{array}$ \\
\hline 9 & No & No & No \\
\hline 10.5 & 14 & 11.77 & 2.25 \\
\hline 12 & 12 & 12.66 & 2.34 \\
\hline 13.5 & 10 & 16.17 & 2.25 \\
\hline 15 & 9 & 19.01 & 2.25 \\
\hline 16.5 & 9 & 20.41 & 2.44 \\
\hline 18 & 8 & 20.07 & 2.44 \\
\hline 19.5 & 7 & 19.37 & 2.25 \\
\hline
\end{tabular}

\subsubsection{Tendency of Formation of Corrugated Pattern}

Once a small sand pile forms on the initially flat surface, the vertical height and lateral position can shift intermittently due to repeated collisions with the attachment of the oscillator. Corrugations formed on the road surface may change in amplitude and wave length with time elapsing. Fig. 9. shows a sample of running spectrum at the track rotating velocity of $18.0 \mathrm{rpm}$ indicating the changes in wave height and its location on with time in the experiment. In this figure, the horizontal coordinate gives the location of the rotating track and the vertical axis stands for the number of rotation. The track used in this experiment is about $157 \mathrm{~cm}$ in circumference and the horizontal axis covers one rotating cycle. When the height of wave is $0 \mathrm{~mm}$, light green is used for plotting. If the height of the wave becomes larger in positive, the part is colored in red. When the height of the wave becomes lower on the contrary, the corresponding portion is colored in blue. The bottom of this figure along the horizontal axis is colored in light green and it shows the wave height is almost flat at the beginning.

As shown in this figure, the initially flat road surface experiences a transient state at the early stage. In this transient state, the motion of the oscillator becomes irregular, and adjacent waves merge and break with each other. After the transient state of first 40 rounds, stable corrugated pattern appears with a constant wave length. As repeating the rotation toward the upward direction along the vertical axis, it can be seen that waves are gradually growing while the convex part of the wave slowly shifts in backward. Finally, regular and constant corrugation emerges on the rotating track. This is a typical example of the experiments, but similar running spectrums are obtained for other cases. Through the experiment, there is a tendency that the faster track rotating velocity is, the more often the transient state occurs, and the transient state tends to take place just after a certain corrugated pattern is well developed.

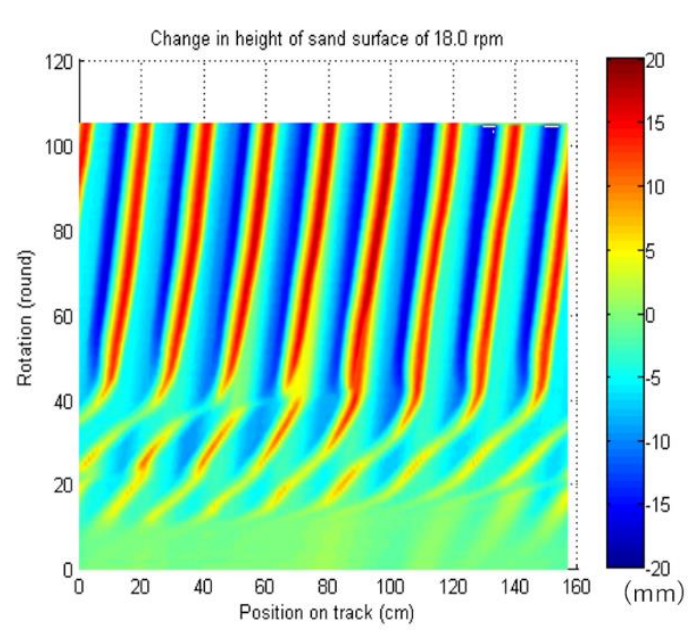

Fig. 9. Running Spectrum of Reproduction Experiment

\subsubsection{Consideration on the Difference in Natural Frequency of Oscillator}

We compared corrugations at the end of the experiment due to the difference in natural frequency of the oscillator. Fig. 10. shows the experimental results on the wave frequency on the road surface when the maximum amplitudes in three types of oscillator were recorded. The natural frequencies of three oscillators were set at $1.37 \mathrm{~Hz}$, $1.46 \mathrm{~Hz}$ and $1.66 \mathrm{~Hz}$. The horizontal axis shows the track rotation velocity and the vertical axis indicates the wave frequency on the road surface at the maximum amplitude. According to this figure, the natural frequency of the oscillator has an effect on the maximum amplitude because smaller natural frequency causes smaller maximum amplitude than others. Fig. 11. shows the experimental results on wave number at the end of the experiment. The horizontal axis stands for the velocity of rotation, and the vertical axis shows the wave number of the corrugation on the track. The smaller a natural frequency of the oscillator is, the smaller the wave number at the end of experiment. As described in 3.2.1, the wave frequency of the road surface at the maximum amplitude is almost constant as approximately $2.3 \mathrm{~Hz}$ to $2.4 \mathrm{~Hz}$ regardless with the natural frequency of the oscillator However, the difference in the natural frequency has an influence on the maximum amplitude of the corrugation. In addition, the growing rate of corrugation is related with the natural frequency of the oscillator and it becomes slower when the natural frequency is smaller.

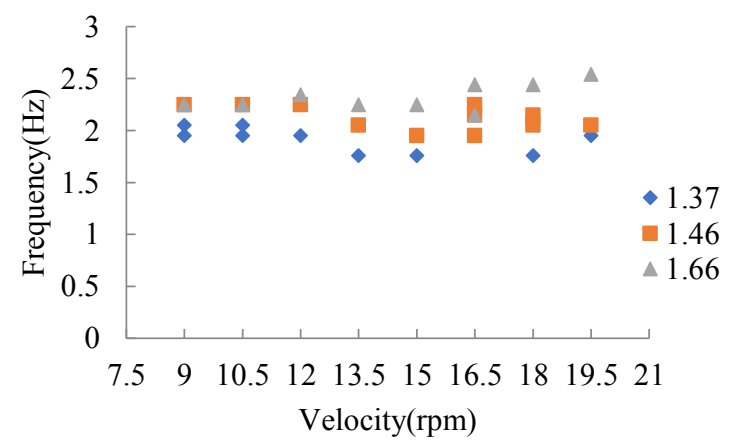

Fig. 10. Relationship between Track Rotation Velocity and Frequency of Road Surface 


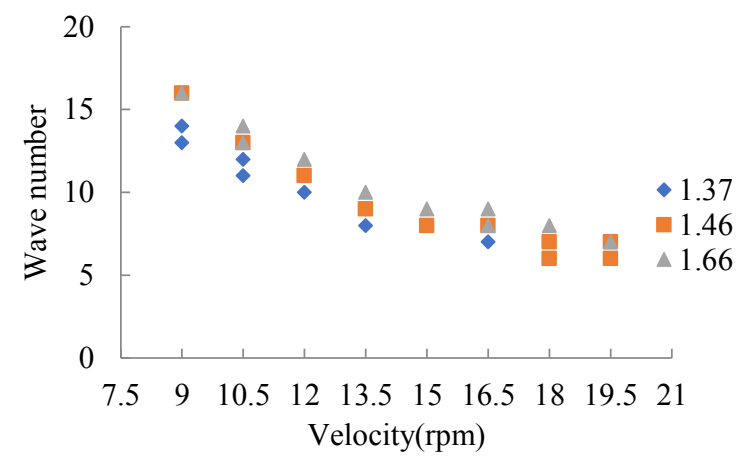

Fig. 11. Relationship between Track Rotation Velocity and Wave Number

\subsection{Difference of the Shape of the Attachment in Reproduction Experiment}

We also conducted the reproduction experiments using different shapes of attachment. In this paper, experimental results of a triangular one with a tip angle of 90 degrees are introduced for comparison with those of a cylindrical one. At the same time, we investigated the effect of moisture content of road material on the corrugation. Table 2. shows the wave number at the end of the experiment of each case. In any road surface condition, it can be said that the triangular attachment is likely to generate corrugation on the road surface because it is more easily penetrable into the surface material than the cylindrical one. On the other hand, spontaneous corrugation is likely to occur under less moist condition of the surface material.

Table 2. Wave Number

\begin{tabular}{|c|c|c|c|c|}
\hline \multirow{2}{*}{\multicolumn{2}{|c|}{ Sand Condition }} & \multirow{2}{*}{$\begin{array}{c}\text { Shape of } \\
\text { Attachment }\end{array}$} & \multicolumn{2}{|c|}{ Velocity(rpm) } \\
\hline & & & 12 & 15 \\
\hline \multirow{2}{*}{\multicolumn{2}{|c|}{ Dry }} & Cylinder & 11 & 8 \\
\hline & & $\begin{array}{l}\text { Triangular } \\
\left(\theta=90^{\circ}\right)\end{array}$ & 10 & 8 \\
\hline \multirow{4}{*}{ Wet } & \multirow[b]{2}{*}{$\begin{array}{c}\text { Around } \\
70 \%\end{array}$} & Cylinder & Slightly & Slightly \\
\hline & & $\begin{array}{l}\text { Triangular } \\
\left(\theta=90^{\circ}\right)\end{array}$ & 13 & 10 \\
\hline & \multirow[b]{2}{*}{$\begin{array}{c}\text { Over } \\
\text { Saturated }\end{array}$} & Cylinder & No & No \\
\hline & & $\begin{array}{l}\text { Triangular } \\
\left(\theta=90^{\circ}\right)\end{array}$ & 13 & 9 \\
\hline
\end{tabular}

\section{Conclusion}

We investigated the spontaneous corrugation patterns of dry sand surface on the self-rotating track formed by the oscillator in indoor experiences. First, we focused on the contact between the wheel and the road surface. It was confirmed by the penetration test that there is a linear relationship between the vertical displacement driven into the test material and the force applied from the attachment. Due to the loading, dry sand deforms in inelastic range and the shape effect of the attachment was also found since the penetration depth differs depending on the difference in a contact angle between the attachment and the test material.
Through the indoor reproduction experiments, it was found that the track rotating velocity and the natural frequency of the oscillator are greatly related to the occurrence of spontaneous corrugations. As the velocity increases, corrugations are formed and it is easy to grow large. Moreover, it was found that the wave frequency of the formed corrugation on the road surface converges where it does not coincide with the natural frequency of the oscillator. Here, we introduced the experimental results with sand material mainly but various conditions of the surface material obviously affect the formation and the growth of corrugation. The moisture content of the surface material is thought as one of those conditions, for example. The authors are conducting the experiments under various conditions including different surface materials such as artificial snow. As a future prospect, we are considering a mathematical model to simulate the formation of spontaneous corrugation patterns.

This work was supported by KAKENHI Grants No. 16K01278.

\section{References}

1. N. Taberlet, S. Morris and J. McElwaine, Physical Review Letters 99, 068003 (2007)

2. Y. Matsukura, A. Kawamura, S. Nakashima and K. Tomiyama, Proceedings of Hokkaido Chapter of the Japan Society of Civil Engineers, 65, E-4 (2008)

3. S. Kanie, H. Zheng, D. Yokomise, Y. Kaneda, Y. Nagata and S. Masaki, Proceedings of Hokkaido Chapter of the Japan Society of Snow and Ice, Annual Report on Snow and Ice Studies in Hokkaido, 35, 5558 (2016)

4. H. Takeshita and K. Kobayashi, Road Engineering \& Management Reveiw, 54, 69-71 (1954)

5. D. C. Mays and B. A. Faybishenko, Complex 5, 51$60(2000)$

6. Y. Nagata, Y. Kaneda and M. Tomita, Proceedings of Hokkaido Chapter of the Japan Society of Snow and Ice, Annual Report on Snow and Ice Studies in Hokkaido, No.32, pp.80-83 (2013)

7. A. Ikeda, T. Srimahachota, H. Zheng and S. Kanie, Proceedings of Hokkaido Chapter of the Japan Society of Civil Engineers, No.73, A-21 (2017)

8. A. Ikeda, T. Srimahachota, H. Zheng and S. Kanie, Proceedings of the 72nd Japan Society of Civil Engineers Annual Meeting, 1-364 (2017)

9. K. Nakarai and T. Yoshida, Soil Found. 55, 857 (2015)

10. T. Srimahachota, H. Zheng, M. Sato and S. Kanie, Proceedings of Hokkaido Chapter of the Japan Society of Civil Engineers, No.73, A-20 (2017)

11. T. Srimahachota, H. Zheng, M. Sato and S. Kanir, Proceedings of the 72nd Japan Society of Civil Engineers Annual Meeting, 1-363 (2017)

12. T. Srimahachota, H. Zheng, M. Sato, S. Kanie and H. Shima, Phys. Rev. E, 96, Issue 6, 062904 (2017) 